\title{
AVALIAÇÃO DA SUSCEPTIBILIDADE MAGNÉTICA USANDO UMA BALANÇA ANALÍTICA
}

\author{
Antonio Adilton O. Carneiro*, Alessandro Tadeu Touso e Oswaldo Baffa \\ Departamento de Física e Matemática, Faculdade de Filosofia Ciências e Letras de Ribeirão Preto, Universidade de São Paulo, Av. \\ Bandeirantes, 3900, 14040-901 Ribeirão Preto - SP
}

Recebido em 7/10/02; aceito em 18/2/03

\begin{abstract}
EVALUATION OF THE MAGNETIC SUSCEPTIBILITY USING AN ANALYTICAL SCALE. A simple system to measure the magnetic susceptibility of different materials is presented. The system uses an analytical scale with sensitivity on the order of micrograms and a permanent NdBFe magnet, based in the Rankine method. In this apparatus, the sample is placed near to the magnet that is fixed on the scale. Depending on the magnetic properties of the sample, an attractive or repulsive force will appear between the magnet and the magnetizing sample. Measuring this force, registered by the scale as a mass, the magnetic parameters such as: permeability and susceptibility of the sample can be determined. Despite it is simplicity the method is quantitative, precise and easily reproducible in many laboratories, what makes it attractive for teaching experiments.
\end{abstract}

Keywords: analytical scale; magnetic susceptibility; magnetic dipole.

\section{INTRODUÇÃO}

A susceptibilidade magnética é uma característica intrínseca de cada material e sua identidade está relacionada com a estrutura atômica e molecular. Os átomos têm momentos de dipolo magnético em virtude do movimento orbital dos respectivos elétrons. Além disso, cada elétron tem um momento de dipolo magnético intrínseco associado ao seu spin. $\mathrm{O}$ momento magnético de um átomo depende da disposição dos elétrons no seu interior. Um material pode produzir um campo magnético tanto porque está magnetizado, como porque conduz uma corrente de transporte de portadores de carga. Quando um material está na presença de um campo magnético, este é modificado por causa da magnetização resultante $\mathbf{M}$ do momento de dipolo molecular. Esta magnetização pode ser puramente devido à interação do campo aplicado com a matéria, conforme ocorre com os materiais diamagnéticos e paramagnéticos ou pode já existir mesmo na ausência do campo externo, conforme ocorre com os materiais ferromagnéticos. $\mathrm{O}$ diamagnetismo ocorre em todos os materiais, pois todas as moléculas exibem um momento de dipolo magnético induzido e antiparalelo ao campo magnético aplicado em virtude da deformação da distribuição da corrente eletrônica. A sua magnetização tende a enfraquecer o campo externo. Geralmente o efeito diamagnético nos materiais é mascarado pelo comportamento paramagnético e ferromagnético. O paramagnetismo resulta da tendência dos momentos magnéticos moleculares alinharem-se com o campo magnético aplicado, reforçando o campo aplicado. Esses materiais diamagnéticos e paramagnéticos têm uma susceptibilidade, em módulo, muito menor que um $(\chi<<1)$.

Rankine apresentou em 1934, um sistema capaz de identificar se a amostra era paramagnética ou diamagnética, através da força de interação magnética entre um ímã permanente e a própria amostra ${ }^{1}$. Quase sessenta anos depois, com o advento dos pequenos e intensos ímãs permanentes de terras-raras, Davis apresentou um sistema, usando o mesmo princípio físico de Rankine mas com arranjo instrumental diferente, capaz de identificar tanto a propriedade magnética da

*e-mail: adilton@dfm.ffclrp.usp.br amostra, bem como quantificar a sua susceptibilidade magnética com variações da ordem da susceptibilidade da água $\left(9,05510^{-6} \mathrm{SI}\right)$. O sistema susceptométrico, descrito por Davis, consiste no uso de uma balança de precisão $(1 \mu \mathrm{g})$ para medir a força de interação magnética entre o ímã permanente e a própria amostra² .

Este trabalho apresenta um método de se medir a susceptibilidade magnética de materiais diamagnéticos e paramagnéticos usando o mesmo princípio físico e instrumental apresentado por Davis, porém com algumas simplificações ${ }^{3}$. A dedução das expressões que relacionam a susceptibilidade magnética com a variação de massa medida na balança é apresentada com detalhes e um novo método é apresentado para se obter a susceptibilidade a partir destas medidas.

\section{MATERIAIS E MÉTODOS}

No aparato instrumental, o volume da amostra é usualmente muito maior que o volume do ímã permanente usado para magnetizar a mesma. Neste caso, o magneto pode ser aproximado por um dipolo de momento magnético $\mathbf{m}$. E, como o ímã fica bastante próximo da amostra, a força de interação magnética entre eles pode ser resolvida usando-se o método das imagens de um dipolo na presença de um plano infinito. Sendo assim, nos cálculos apresentados a seguir, a amostra magnetizada será substituída por um dipolo equivalente $\mathbf{m}_{\mathbf{e}}$ sobre o mesmo eixo de simetria do dipolo magnético do ímã e separado por distância equivalente a duas vezes a distância entre o centro do ímã e a superfície da amostra, conforme ilustra a Figura $1^{4,5}$.

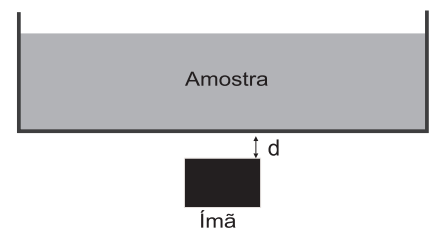

B

Dipolo equivalente $(\bar{m})$

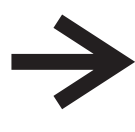

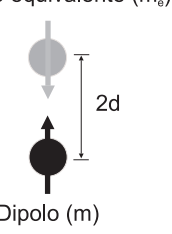

Figura 1. Ilustração esquemática da substituição da amostra por um dipolo equivalente: a) magnetização da amostra pelo ímã e b) aplicação do método de imagens entre um dipolo magnético e um plano 
O campo magnético $\vec{B}$ num ponto $\mathbf{P}$ qualquer gerado por um dipolo localizado na origem do sistema de coordenadas pode ser descrito pela seguinte relação:

$$
B=\frac{\mu_{0}}{4 \pi} \frac{1}{r^{3}}[3 \cdot(\vec{m} \cdot \hat{r}) \hat{r}-\vec{m}]
$$

sendo $\mu_{0}$ a permeabilidade no vácuo, $\vec{m}$ o momento magnético do ímã e $\vec{r}$ a distância entre o ímã e a amostra. $\left(\mu_{\mathrm{o}}=4 \pi \cdot 10^{-7} \mathrm{NA}^{-2}\right.$, no $\mathrm{SI})$.

Como o dipolo está orientado na direção $z$ que é a mesma direção de $\vec{r}$, temos que $\vec{m} \cdot \hat{r}=m$, então a Equação 1 se reduz a:

$B=|\vec{B}|=\frac{\mu_{0}}{4 \pi \cdot r^{3}}[2 m]$

A força magnética $\vec{F}_{m}$ entre dois dipolos magnéticos é definida como sendo igual a

$\vec{F}_{m}=\left(\vec{m}_{1} \nabla\right) \cdot \vec{B}_{2}$

sendo $\vec{m}_{1}$ o momento magnético do dipolo 1 e $\vec{B}_{2}$ o campo magnético gerado pelo dipolo 2 .

Substituindo a Equação 2 na Equação 3 e considerando o dipolo 1 e 2 como sendo o dipolo do ímã e o dipolo equivalente, conforme mostra a Figura 1, tem-se:

$\vec{F}=\vec{m} \cdot \nabla\left(\frac{\mu_{0} \cdot \vec{m}_{e}}{2 \pi \cdot z^{3}}\right)$

$\vec{F}=\vec{m} \cdot \frac{\partial}{\partial z} \frac{\mu_{0} \vec{m}_{e}}{2 \pi \cdot z^{3}}=\frac{3 \mu_{0} \vec{m} \cdot \vec{m}_{e}}{2 \pi \cdot z^{4}}$

sendo $z$ a distância entre os dois dipolos.

Substituindo a distância entre os dois dipolos pela distância entre o ímã e a amostra $(z=2 \mathrm{~d})$ e tirando o módulo da força tem-se:

$F=\frac{3 \mu_{0} m \cdot m_{e}}{32 \pi d^{4}}$

Sendo a magnetização da amostra devido à presença do ímã uma relação entre o momento equivalente $m_{e}$ e o momento do ímã $m$ pode ser obtida aplicando a lei de Gauss e a lei circuital de Ampére, nas condições de contorno sobre os vetores de campo magnético na interface entre a amostra e o ar $^{6}$. Fazendo isto tem-se:

$m_{e}=\frac{\mu_{r}+1}{\mu_{r}-1} m$

sendo $\mu_{r}$ a permeabilidade magnética da amostra $(\mu)$ relativa à do ar $\left(\mu_{o}\right)$, ou seja, $\mu_{r}=\mu / \mu_{o}$. Por definição, $\mu_{r}=1+\chi$, sendo $\chi$ a susceptibilidade volumétrica da amostra. Reescrevendo a Equação 7 em função da susceptibilidade $\chi$ tem-se:

$m_{e}=\frac{\chi}{2+\chi} m$

Substituindo a Equação 8 na Equação 6, temos que a força de interação entre o ímã e a amostra é representada por:

$F=\frac{3 \mu_{o}}{64 \pi} \frac{m^{2}}{d^{4}} \frac{\chi}{(1+\chi / 2)}$
As amostras que permitem ser avaliadas com esta técnica deverão ser paramagnéticas ou diamagnéticas, isto é $\chi<<1$. Logo, o termo $(1+\chi / 2)$ da Equação 9 pode ser aproximado por 1 , ou seja:

$F=\frac{3 \mu_{o} m^{2} \chi}{64 \pi d^{4}}$

Quando a amostra é exposta ao campo magnético produzido pelo ímã permanente, ela experimenta uma força de interação que será atrativa no caso paramagnético $(\chi>0)$ e repulsiva quando a amostra for diamagnética $(\chi<0)$. Esta força magnética é registrada como sendo igual à força peso medida através da balança e é linearmente proporcional à susceptibilidade da amostra, conforme mostra a Equação 10.

Na ausência da amostra, o meio material presente é o ar. Portanto, ao adicionar a amostra, um mesmo volume do ar é deslocado. Sendo assim, a contribuição para a força será devido à presença da amostra subtraída da contribuição do ar removido, ou seja,

$F_{\text {total }}=F_{\text {amostra }}-F_{a r}=\frac{3 \mu_{0}}{64 \pi} \frac{m^{2}}{d^{4}}\left(\chi-\chi_{a r}\right)$

sendo $\chi_{a r}$ o valor da susceptibilidade volumétrica do ar $\left(\chi_{a r}=0,364 \mathrm{x}\right.$ $10^{-6}$, no SI).

A variação da força peso $(\Delta P)$ registrada na balança, quando a amostra está presente, é igual a força magnética de interação entre o ímã e a amostra, ou seja,

$\Delta P=m_{o} \cdot g=F_{\text {total }}$

sendo que $m_{o}$ tem unidade de massa e é obtida da leitura direta da balança e $g$ é a aceleração da gravidade.

Igualando as Equações 11 e 12 tem-se:

$\chi=\chi_{a r}+\frac{m_{0} g 64 \pi d^{* 4}}{3 \mu_{0} m^{2}}$

onde $d^{*}$ representa a verdadeira distância entre o centro do ímã e a amostra, quando esta estiver presente e é igual a:

$d^{*}=d+\delta d$

sendo $d$ a distância entre o centro do ímã e a amostra padrão, ou seja, amostra com susceptibilidade conhecida, e $\delta d$ a variação no deslocamento do prato da balança, devido à presença de amostra em estudo relativo à sua posição quando com a amostra padrão. Esta variação $\delta d$ está relacionada com a massa $\left(m_{o}\right)$ registrada na balança pela seguinte relação:

$\delta d=-0,22 *\left(m_{o, \text { amostra }}-m_{o, \text { ref }}\right)$

sendo $m_{\text {o,amostra }}$ a leitura na balança com a amostra em estudo e $m_{\text {o,ref }}$ com a amostra padrão (referência). Considerou-se $m_{o}$ em $K g$ e $d$ em metros.

As Equações 12-15 permitem determinar o valor aproximado da susceptibilidade magnética volumétrica através de medidas diretas na própria amostra em estudo. Mas para isto, é preciso que se tenha também o valor preciso da distância entre a superfície da amostra e a do ímã $\left(d^{*}\right)$, bem como o valor do momento magnético do ímã (m). Considerando que este último seja conhecido ou pelas informações do fabricante ou determinado por métodos precisos de medidas magnéticas, a distância $d$ pode ser determinada através de medidas em amostras conhecidas sendo que, neste trabalho por exemplo, usamos água pura $\left(\chi=9,03210^{-6} \mathrm{SI}\right)$ como referência. 
Uma vez que a susceptibilidade varia com a quarta potência da distância e o quadrado do momento magnético, conforme mostra a Equação 13, pequenos erros na determinação destes parâmetros provocam grandes erros na medida da susceptibilidade magnética. Para tanto, uma forma indireta de se determinar a susceptibilidade magnética volumétrica da amostra de interesse, usando o mesmo arranjo instrumental descrito no trabalho de Davis, é apresentada a seguir.

Quando diferentes materiais estão misturados homogeneamente no mesmo volume, a susceptibilidade magnética da mistura é definida como sendo igual a:

$\chi=\sum_{i} c_{i} \chi_{i}$

sendo $c_{i}$ e $\chi_{\mathrm{i}}$ a concentração e a susceptibilidade mássica do material presente no volume total da amostra.

Portanto, se misturarmos uma porção da amostra de interesse com um outro material de susceptibilidade bem conhecida tem-se que a susceptibilidade do composto é igual a:

$\chi=c_{a} \chi_{m, a}+c_{s} \chi_{m, s}$

sendo $c_{a}$ e $\chi_{a}$ a concentração e a susceptibilidade mássica do material de interesse e $c_{\mathrm{s}}$ e $\chi_{\mathrm{s}}$ a concentração e a susceptibilidade mássica do solvente.

Igualando a Equação 13 com a Equação 17 tem-se que a força peso $\left(m_{\sigma} \cdot g\right)$ é diretamente proporcional às concentrações dos materiais presentes no composto. Fazendo medidas em duas ou mais amostras deste composto, de mesmo volume mas com diferentes concentrações, e traçando-se uma curva que ajusta os pontos $\left(m_{o} x c_{a}\right)$, podese a partir desta curva determinar os valores de $m_{o}$ para qualquer concentração.

De acordo com a Equação 13, quando a variação da força peso registrada na balança for nula, ou seja $m_{0}=0$, a susceptibilidade da amostra será igual à do próprio ar. Encontrando na curva o valor da concentração para este ponto onde $m_{o}=0$, ou seja o ponto de interseção da curva com o eixo que representa a concentração, e substituindo na Equação 16, temos que o valor da susceptibilidade da amostra em estudo será dado por:

$\chi_{m, a}=\left(\chi_{a r}-c_{s, m_{0}=0} \chi_{m, s}\right) / c_{a, m_{o}=0}$

sendo $c_{s, m_{o}=0}$ e $c_{a, m_{o}=0}$ a concentração do solvente e da amostra (soluto) para a condição da força peso registrada na balança ser nula.

\section{ASPECTOS EXPERIMENTAIS}

O sistema montado para as realizações das medidas apresentadas a seguir está ilustrado na Figura 2. Este consiste de uma balança analítica (marca: MLW, modelo: mp20) composta de dois pratos, um pequeno ímã usado como fonte de magnetização e um portaamostras. Em um dos pratos foi colocado um pequeno ímã preso por um suporte. Sobre o ímã, mas ligeiramente separado do mesmo e desacoplado da balança, foi posto o porta-amostra cilíndrico com $0,0528 \mathrm{~m}$ de diâmetro e $0,035 \mathrm{~m}$ de altura. Sobre o outro prato foi posto um contrapeso com massa ligeiramente maior que a soma da massa do ímã e do seu suporte de modo que a balança fique no equilíbrio num ponto intermediário da sua escala de medida (peso $\cong$ fundo de escala/2) quando na ausência da amostra. A escolha deste ponto de equilíbrio deslocado do zero é para que possamos medir tanto amostras paramagnéticas quanto diamagnéticas.

Para reduzir interferências de vibrações, todo o sistema foi posto dentro de uma caixa de acrílico e posicionado sobre uma superfície bastante rígida. A balança tem uma capacidade máxima para $20 \mathrm{~g}$ e

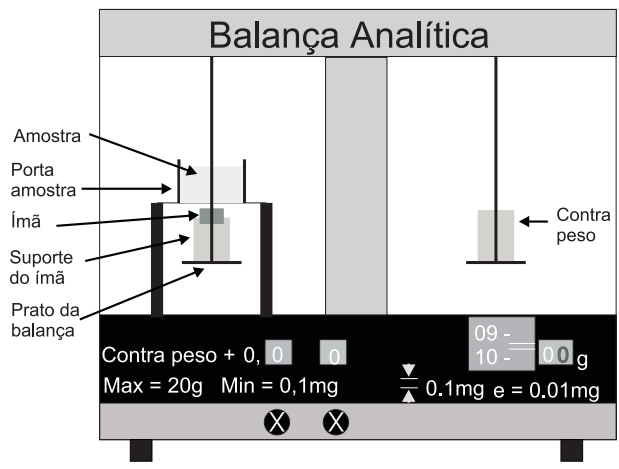

Figura 2. Diagrama esquemático do sistema desenvolvido para medir a susceptibilidade magnética de diferentes amostras

sua sensibilidade é de $10 \mu \mathrm{g}$. O ímã permanente utilizado é de Neodímio-Ferro-Boro que gera um campo magnético em torno de 3300 Gauss próximo à sua superfície e tem um momento magnético $(m)$ de aproximadamente 0.1905 A.m ${ }^{2}$. Ele é de formato cilíndrico com $0,00510 \mathrm{~m}$ de altura e $0,0064 \mathrm{~m}$ de diâmetro. No arranjo apresentado, a distância $d^{*}$ entre a amostra e o ímã pode variar de 0 a $0,005 \mathrm{~m}$. O valor a ser escolhido depende da força de interação entre o ímã e a amostra e da propriedade magnética da mesma. No caso em que a amostra for paramagnética a força será repulsiva, logo a distância entre o porta-amostra e o ímã deve ser mínima. Por outro lado, se amostra for diamagnética, a força será atrativa, logo a distância entre o porta-amostra deve ser tal que o ímã não encoste no mesmo quando na presença da amostra. No primeiro caso, o equivalente em massa registrado pela balança $\left(m_{o}\right)$ é atribuído positivo e no segundo caso é atribuído negativo.

\section{Procedimentos de medidas}

O equivalente em massa $\left(m_{o}\right)$ registrado na balança é atribuído como sendo a diferença da medida realizada antes e após a presença da amostra que deverá ser colocada no porta-amostra, com o máximo de cuidado. As amostras em forma de soluções deverão ser adicionadas com a ajuda de uma seringa acoplada ao porta-amostra, através de um tubo flexível. No caso de amostras sólidas, estas deverão ser colocadas com o máximo de cuidado sem tocar nas plataformas da balança. Neste caso, as medidas feitas em soluções oferecem maiores precisões do que aquelas em sólidos, devido às menores interferências causadas durante o manuseio e a compactação como nas amostras em pó.

\section{Avaliação do sistema}

Medidas da susceptibilidade foram realizadas em soluções de $\mathrm{Fe}^{3+}$ e $\mathrm{Mn}^{2+}$ com diferentes concentrações. Estas foram preparadas misturando $\mathrm{FeCl}_{3} \cdot 6 \mathrm{H}_{2} \mathrm{O}$ e $\mathrm{MnSO}_{4} \cdot \mathrm{H}_{2} \mathrm{O}$ em água à uma concentração de $0-15 \mathrm{mg} / \mathrm{g}$. Para cada medida, $20 \mathrm{ml}$ de amostra era colocado dentro do porta-amostra. Foi realizada uma série de cinco medidas para cada amostra e depois calculada a média. A Figura 3 mostra o equivalente em massa $\left(m_{o}\right)$ registrada na balança versus a concentração de soluto nas duas soluções. A pequena dispersão observada no ajuste linear desses pontos está relacionada com a variação na distancia $d^{*}$ entre o ímã e a amostra para as diferentes concentrações de soluto, ou seja, para cada registro na balança tem-se um valor de $d^{*}$ associado (ver Equações 14 e 15). E, de acordo com as Equações 13 e 14 , a concentração varia com a quarta potência de $d^{*}$. A transição do efeito diamagnético $\left(\mathrm{m}_{\mathrm{o}}\right.$ negativo) para o paramagnético $\left(\mathrm{m}_{\mathrm{o}}\right.$ po- 
sitivo) também pode ser nitidamente observada nas duas curvas da Figura 3.
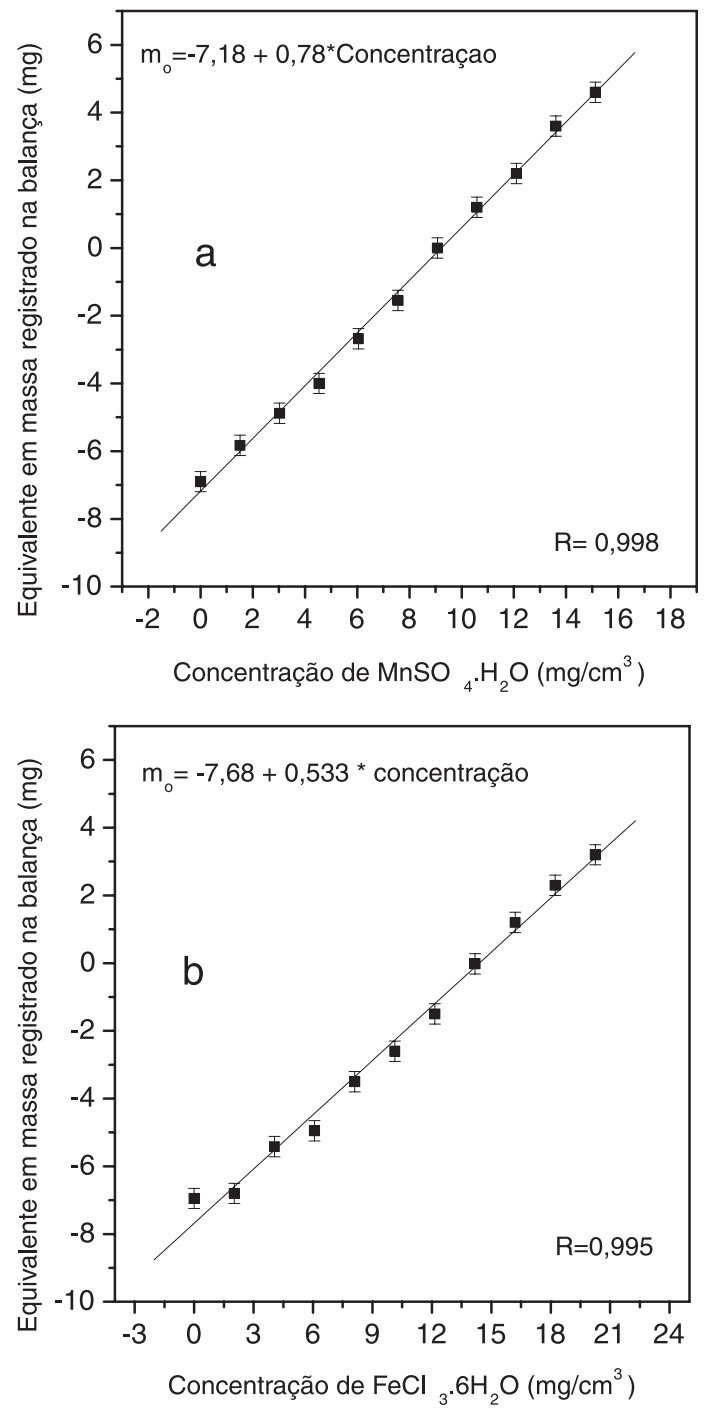

Figura 3. Variação da força peso $\left(m_{o}\right)$ medida na balança versus concentração mássica de: a) $\mathrm{FeCl}_{3} \cdot 6 \mathrm{H}_{2} \mathrm{O}$ e b) $\mathrm{MnSO}_{4} \cdot \mathrm{H}_{2} \mathrm{O}$ na solução

A Tabela 1 mostram os valores da susceptibilidade volumétrica das soluções $\left(\chi_{s}\right)$ de $\mathrm{FeCl}_{3} \cdot 6 \mathrm{H}_{2} \mathrm{O}$ e de $\mathrm{MnSO}_{4} \cdot \mathrm{H}_{2} \mathrm{O}$, bem como as susceptibilidades mássicas destes solutos $\left(\chi_{m, s}\right)$, determinadas através das Equações 13-17, a distância $d$ entre o ímã e a amostra de referência foi determinada através da medida em água pura $\left(\chi=9,032 \times 10^{-6}\right)$ e cujo valor obtido foi de $9,792 \times 10^{-4} \mathrm{~m}$. Os valores médios da susceptibilidade mássica obtida para o $\mathrm{FeCl}_{3} \cdot 6 \mathrm{H}_{2} \mathrm{O}$ e o $\mathrm{MnSO}_{4} \cdot \mathrm{H}_{2} \mathrm{O}$, a partir da Tabela 1 , foram $0,62 \pm 0,06 \times 10^{-6}$ e $1,07 \pm 0,01 \times 10^{-6}$, respectivamente. Os valores obtidos a partir das curvas da Figura 3 e da Equação 15 foram de $0,66 \pm 0,05 \mathrm{~m}^{3} / \mathrm{Kg}$ e $1,01 \pm 0,05 \mathrm{~m}^{3} / \mathrm{Kg}$. Esses valores estão de acordo com os tabelados para estes materiais $\left(0,709 \mathrm{~m}^{3} / \mathrm{Kg} \text { e } 1,05 \mathrm{~m}^{3} / \mathrm{Kg}\right)^{7}$. O ponto referente à água pura na Figura $3 \mathrm{~b}\left(c_{f e}=0\right)$ encontra-se um pouco fora da curva. Acredita-se que esta distorção se deve a uma possível auto-oxidação do ferro em meio aquoso.

Nos dois modelos usados para a determinação da susceptibilidade magnética, conforme apresentado acima, não foi necessária a medida direta da distância $d^{*}$, evitando assim maiores dispersões na determinação da susceptibilidade por causa da sua dependência com
Tabela 1. Apresentação das concentrações de $\mathrm{FeCl}_{3} \cdot 6 \mathrm{H}_{2} \mathrm{O}$, da força peso $\left(\mathrm{m}_{\mathrm{o}}\right)$ registradas na balança, da susceptibilidade volumétrica da solução $\left(\chi_{s}\right)$ e da susceptibilidade mássica do soluto $\left(\chi_{m, s}\right)$ determinada pelas Equações 12-17

\begin{tabular}{|c|c|c|c|}
\hline $\begin{array}{l}\text { Concentração } \\
\qquad\left(\mathrm{Kg} / \mathrm{m}^{3}\right)\end{array}$ & $\begin{array}{l}\mathrm{m}_{\mathrm{o}} \\
(\mathrm{mg})\end{array}$ & $\chi$ da solução $\left(10^{-6}\right)$ & $\begin{array}{c}\chi_{\mathrm{m}, \mathrm{s}} \text { do soluto }\left(10^{-6}\right) \\
\left(\mathrm{m}^{3} / \mathrm{Kg}\right)\end{array}$ \\
\hline \multicolumn{4}{|c|}{ Soluto $-\mathrm{FeCl}_{3} \cdot 6 \mathrm{H}_{2} \mathrm{O}$} \\
\hline 0 & $-0,695$ & $-9,032$ & - \\
\hline 2,02 & $-0,630$ & $-7,906$ & 0,56 \\
\hline 4,05 & $-0,542$ & $-6,472$ & 0,63 \\
\hline 6,07 & $-0,495$ & $-5,747$ & 0,54 \\
\hline 8,10 & $-0,350$ & $-3,677$ & 0,66 \\
\hline 10,12 & $-0,260$ & $-2,515$ & 0,64 \\
\hline 12,15 & $-0,150$ & $-1,214$ & 0,64 \\
\hline 14,17 & 0,002 & 0,379 & 0,66 \\
\hline \multicolumn{4}{|c|}{$\bar{\chi}_{m, s} \pm \Delta \chi_{m, s}=0,62 \pm 0,06$} \\
\hline \multicolumn{4}{|c|}{ Soluto - $\mathrm{MnSO}_{4} \cdot \mathrm{H}_{2} \mathrm{O}$} \\
\hline 0 & $-0,695$ & $-9,032$ & - \\
\hline 1,512 & $-0,583$ & $-7,120$ & 1,25 \\
\hline 3,024 & $-0,488$ & $-5,641$ & 1,12 \\
\hline 4,536 & $-0,400$ & $-4,362$ & 1,02 \\
\hline 6,048 & $-0,268$ & $-2,614$ & 1,06 \\
\hline 7,56 & $-0,155$ & $-1,27$ & 1,02 \\
\hline 9,072 & 0 & 0,360 & 1,03 \\
\hline 10,584 & 0,120 & 1,464 & 0,99 \\
\hline \multicolumn{4}{|c|}{$\bar{\chi}_{m, s} \pm \Delta \chi_{m, s}=1,07 \pm 0,01$} \\
\hline
\end{tabular}

a quarta potência em $d^{*}$. Embora o desvio no parâmetro $m_{o}$ registrado na balança seja indicado pelo fabricante como sendo igual a 0,01 mg, o desvio padrão médio obtido, para cinco medidas repetidas por amostra, foi de aproximadamente $0,005 \mathrm{mg}$. Para a medida feita na referência (água) este desvio correspondeu a um erro relativo de aproximadamente $1 \%$. Este pequeno desvio na indicação da balança foi obtido minimizando a vibração da mesma e a presença de materiais magnéticos em movimento ao seu redor. Considerando que o erro relativo no valor do momento magnético do ímã, indicado pelo fabricante, é de $1 \%$, o erro relativo propagado na determinação do parâmetro $d$ através da Equação 13 é de aproximadamente 1,5\% e o erro relativo propagado na determinação da susceptibilidade mássica $\chi_{m}$ das amostras analisadas, usando as Equações 13 e 17 foi de, aproximadamente, $8 \%$.

Estes resultados confirmam a possibilidade de aplicação de uma simples técnica para a medida da susceptibilidade magnética de materiais.

\section{CONCLUSÕES E PERSPECTIVAS}

As medidas da susceptibilidade realizadas com a balança de sensibilidade moderada e de fácil uso apresentaram resultados que confirmam a possibilidade de uso de uma técnica simples para medida da susceptibilidade em amostras diamagnéticas ou paramagnéticas de baixa concentração. Como a medida é feita com o magneto sempre fixo sobre o prato da balança e desacoplado do porta-amostra, o equivalente em massa registrado é devido apenas à força magnética entre o magneto e a amostra magnetizada pelo próprio magneto. Sendo assim, qualquer possível interação magnética entre o magneto e a balança não interferirá na medida. Além disso, geralmente, os pratos das balanças (analíticas ou digitais), usadas em laboratórios de química, são de aço inox, ou seja, são não magnetizáveis. 
Neste aparato instrumental, o equipamento mais dispendioso é a balança que normalmente está disponível em laboratórios de química tornando o método bastante atrativo para experimentos de ensino, uma vez que tópicos de magnetismo aplicado à química são pouco explorados do ponto de vista experimental pela dificuldade instrumental.

\section{AGRADECIMENTOS}

Os autores agradecem à Fapesp, ao Capes e ao CNPq pelos auxílios concedidos e ao Sr. L. Rocha pelo apoio técnico.

\section{REFERÊNCIAS}

1. Rankine, A. O.; Proc. Phys. Soc. 1934, 46, 391.

2. Davis, R. S.; Am. J. Phys. 1992, 60, 365.

3. Davis, R. S.; Meas. Sci. Technol. 1993, 4, 141.

4. Griffiths, D. J. Em Introduction to Electrodynamic; Reeves, A.; Dellas, K., eds.; $3^{\text {rd }}$ ed., Prentice Hall, 1999, p. 121.

5. Tipler, P. A.; Eletricidade e Magnetismo, 1995, vol. 3, p. 261.

6. Reitz, J. R.; Milford, F. J.; Christy, R. W.; Fundamentos da Teoria Eletromagnética, ed. Campus, 1991, p. 200.

7. Weast, R.; Handbook of Chemistry and Physics, E-129. 